\title{
Expression profiling of microRNAs in optineurin (E50K) mutant transgenic mice
}

\author{
LIN GAO, BO JIANG, DAWEI LEI, XINRONG ZHOU and HUIPING YUAN \\ Department of Ophthalmology, The Second Affiliated Hospital, Harbin Medical University, \\ Nangang, Harbin 150086, P.R. China
}

Received February 2, 2015; Accepted May 18, 2015

DOI: $10.3892 /$ br.2015.565

\begin{abstract}
An E50K substitution in the transcription factor optineurin $(O P T N)$ induces primary open-angle glaucoma (POAG). To explore the potential role of microRNAs (miRNAs) in E50K OPTN-induced POAG, miRNA expression profiling was performed on retinal samples from OPTN (E50K) transgenic and wild-type mice. The retinas were collected from 30 transgenic and 30 wild-type mice, and miRNA expression was evaluated using a genome-wide miRNA microarray. miRNAs that were differentially expressed in retinal samples from OPTN (E50K) transgenic mice were identified and validated by reverse transcription-quantitative polymerase chain reaction (RT-qPCR). Additional gene ontology and signaling pathway analyses were performed using bioinformatics tools. A total of 48 miRNAs exhibited increased or decreased expression in the retinas from OPTN (E50K) transgenic mice when compared with the expression in the retinas from wild-type mice. A total of 5 miRNAs with increased expression in OPTN (E50K) transgenic mice could be grouped into one cluster as they belong to the miR- 8 family and may act as regulators in the development of POAG in OPTN (E50K) transgenic mice. RT-qPCR results confirmed significantly increased expression of miR-141 in the retinas of OPTN (E50K) transgenic mice as compared to wild-type mice. In conclusion, these results show that certain miRNAs are differentially expressed in the retinas of OPTN (E50K) transgenic mice and may play roles in the pathogenesis of POAG induced by $O P T N(\mathrm{E} 50 \mathrm{~K})$.
\end{abstract}

\section{Introduction}

Glaucoma causes irreversible blindness and is characterized by optic nerve degeneration and visual field defects (1). Primary

Correspondence to: Professor Huiping Yuan, Department of Ophthalmology, The Second Affiliated Hospital, Harbin Medical University, 246 Xuefu Road, Nangang, Harbin 150086, P.R. China E-mail: yuanhp2013@126.com

Key words: optineurin (E50K), mutation, microarray, microRNA, retinal ganglion cells apoptosis open-angle glaucoma (POAG) is the major form of glaucoma. An epidemiological study in Japan demonstrated that a large number of POAG cases were diagnosed as normal tension glaucoma (NTG) (2). Mutations in the optineurin (OPTN) gene that encodes amino acid substitutions, such as E50K, H486R and R545Q, have been associated with POAG and NTG (3-6). The substitution of glutamic acid by lysine at amino acid 50 $(\mathrm{E} 50 \mathrm{~K})$ is exclusively associated with familial and sporadic forms of NTG (7). Previous studies have shown that E50K OPTN induces the apoptosis of retinal ganglion cells in transgenic mice models and progressive retinal degeneration exclusively in the peripheral region of the retinas, although the exact mechanism remains unclear (8).

MicroRNAs (miRNAs) bind to target messenger RNAs (mRNAs) and subsequently suppress protein expression via mRNA degradation or translational inhibition (9). Several reports have described the expression of miRNAs in the eye and specific miRNAs have been indicated in the pathogenesis of glaucoma. In one report, miRNA-24 was shown to regulate transforming grow th factor $\beta 1$ processing during cyclic mechanical stress in human trabecular network cells (10). It is unclear whether miRNAs contribute to pathogenesis in OPTN (E50K) transgenic mice. To address whether additional miRNAs could play a role in OPTN (E50K)-induced POAG pathogenesis, miRNA profiling was performed of the retinal samples from OPTN (E50K) transgenic mice and the differentially expressed miRNAs were identified. Differential expression of miRNAs was validated by reverse transcription-quantitative polymerase chain reaction (RT-qPCR). The identification of the differentially expressed miRNAs due to mutant OPTN (E50K) expression is likely to provide a foundation for studying the molecular pathways contributing to POAG.

\section{Materials and methods}

Animals and sample collection. All the animal experiments in the study were performed according to the tenets of the National Institutes of Health Guidelines and Regulations on the Care and Use of Animals in Research. Transgenic mice engineered to express the E50K mutant human OPTN in the retina were as described previously (11). Retinas from 8-month-old transgenic and wild-type mice were collected for genome-wide miRNA expression analysis. Retinas from 3 groups of 10 transgenic mice (E1, E2 and E3) and 3 groups of 
10 wild-type mice (W1, W2 and W3) were pooled for analysis and independently analyzed for miRNA expression. Retinal samples were frozen at $-80^{\circ} \mathrm{C}$ until analysis.

miRNA microarray study. miRNA microarray experiments were performed at CapitalBio Corp. (Beijing, China). Total RNA was extracted with TRIzol reagent (Invitrogen Life Technologies, Carlsbad, CA, USA) from retinal samples collected from transgenic and wild-type mice. Samples were labeled and hybridized to a GeneChip ${ }^{\circledR}$ miRNA Array (V3.0; Affymetrix, Santa Clara, CA, USA). Raw data were normalized and analyzed using the miRNA QC Tool software (Affymetrix). The analysis focused on miRNAs with a $\geq 1.5$-fold expression difference in retinal samples from $O P T N$ (E50K) transgenic mice as compared to wild-type control retinal samples.

miRNA target gene prediction and function analysis. Prediction of miRNA target genes was performed using a computational approach. The prediction of miRNA target genes was performed using 3 different miRNA target prediction algorithms: PicTar, miRanda v5 and TargetScan v6.0. For each algorithm, the potential binding sites in the mRNA 3'-untranslated region (3'-UTR) of target genes were identified according to specific base-pairing rules.

RT-qPCR miRNA analysis. To confirm the results from the miRNA microarray, RT-qPCR analysis of mmu-miR-141 was performed on the same total RNA used for microarray analysis. First-strand cDNA synthesis was performed from equal amounts of total RNA using an All-in-One ${ }^{\mathrm{TM}}$ miRNA First-Strand cDNA Synthesis kit (GeneCopoeia, Guangdong, China) according to the manufacturer's instructions. Primers were designed and synthesized by GeneCopoeia.

RT-qPCR was performed using the All-in-One ${ }^{\mathrm{TM}}$ qPCR Mix on LightCycler 480 system (Roche, Basel, Switzerland). The levels of an endogenous control, U6, were used to normalize the expression levels of each miRNA. All the reactions were performed in triplicate and included controls. The fold-change in miRNA expression was calculated using the comparative $\mathrm{Ct}$ method. Data are presented as the fold-change relative to expression in the retinal samples of wild-type mice.

Statistical analysis. All the results are expressed as the mean \pm standard deviation. Statistical analysis was performed with the Student's t-test to identify statistically significant differences using commercial software (SigmaPlot; Systat Software Inc., San Jose, CA, USA). P $<0.05$ was considered to indicate a statistically significant difference.

\section{Results}

Global miRNA profiling in OPTN (E50K) transgenic and wild-type mice. Retinal miRNA expression patterns were evaluated using pooled retinal samples for 3 separate groups of 10 transgenic mice (E1, E2 and E3) and 10 wild-type mice (W1, W2 and W3). For each pooled sample, $>2 \mu \mathrm{g}$ of retinal RNA was harvested. Spectrophotometric analysis of RNA purity was performed by measuring absorbance at 260 and
A
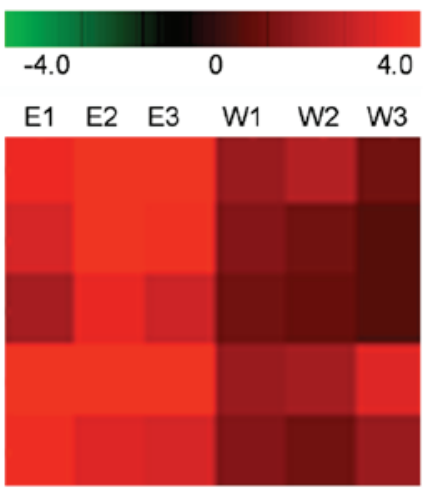

mmu-miR-141_st

mmu-miR-451_st

mmu-miR-3085-3p_st

mmu-miR-483-star_st

mmu-miR-712_st

B

mmu-miR-141_st

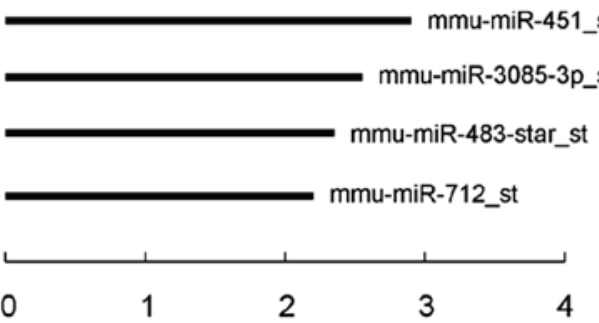

Figure 1. MicroRNA (miRNA) profiles were performed on the retinas of optineurin $(O P T N)$ (E50K) transgenic and wild-type mice. (A) Hierarchical clustering was performed with normalized miRNA data (fold-change $\geq 1.5$ ) that passed the Student's t-test $(\mathrm{P}<0.05)$. A total of 5 miRNAs were identified based on significant expression differences in the retinas of OPTN (E50K) mutation transgenic mice as compared to wild-type mice. E1-E3 and W1-W3 represent miRNA expression data for three independent samples of pooled retinal RNA from OPTN (E50K) transgenic or wild-type mice, respectively. For each miRNA, the intensity of the red color reflects the expression levels. (B) Fold-change in miRNA expression in the retinas of OPTN (E50K) transgenic mice as compared to wild-type mice, determined by miRNA array.

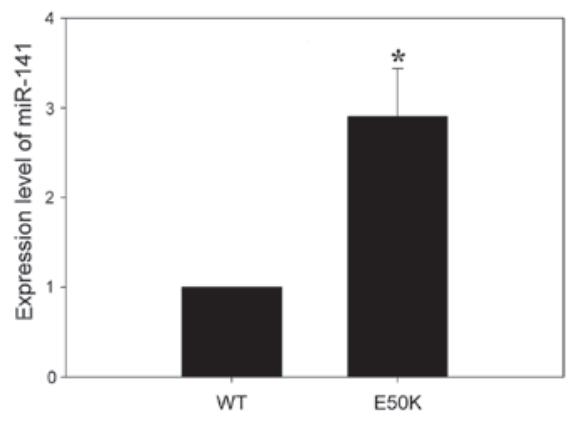

Figure 2. Validation of selected microarray data by reverse transcription-quantitative polymerase chain reaction (RT-qPCR). Relative expression levels of miR-141 were determined by RT-qPCR. mmu-miR-141 expression was markedly upregulated in the retinas of optineurin (E50K) transgenic mice compared to wild-type (WT) mice. Values are means \pm standard deviation and are expressed relative to internal control (U6; $n=3$ for each group; ${ }^{*} \mathrm{P}<0.05$, Student's t-test)

$280 \mathrm{~nm}$. All samples exhibited 260/280 nm absorbance ratios $>1.96$, indicating isolation of high-quality retinal RNA.

Global miRNA expression patterns were determined for each sample by microarray analysis. The analysis focused on specific miRNAs exhibiting differential expression levels in the retinas from OPTN (E50K) transgenic mice as compared to wild-type mice by identifying miRNAs with a $\geq 1.5$-fold-change in expression level and $\mathrm{P} \leq 0.05$. Based on this threshold, 48 miRNAs were differentially expressed in OPTN 
Table I. MicroRNA (miRNA) profiles were performed on the retinas of optineurin (E50K) transgenic and wild-type mice. miRNAs are ranked in order of fold-change, from the largest to the smallest.

\begin{tabular}{|c|c|c|}
\hline miRNA ID & Fold-change & miRNA sequence \\
\hline mmu-miR-141 & 3.2023 & UAACACUGUCUGGUAAAGAUGG \\
\hline mmu-miR-451 & 2.8473 & AAACCGUUACCAUUACUGAGUU \\
\hline mmu-miR-200a & 2.6426 & UAACACUGUCUGGUAACGAUGU \\
\hline mmu-miR-483* & 2.5317 & UCACUCCUCCCCUCCCGUCUU \\
\hline mmu-miR-3057-3p & 2.4620 & UCCCACAGGCCCAGCUCAUAGC \\
\hline mmu-miR-877* & 2.4260 & UGUCCUCUUCUCCCUCCUCCCA \\
\hline mmu-miR-3102-3p.2 & 2.4235 & CUCUACUCCCUGCCCCAGCCA \\
\hline mmu-miR-429 & 2.3851 & UAAUACUGUCUGGUAAUGCCGU \\
\hline mmu-miR-3085-3p & 2.3604 & UCUGGCUGCUAUGGCCCCCUC \\
\hline mmu-miR-712 & 2.3241 & CUCCUUCACCCGGGCGGUACC \\
\hline mmu-miR-223 & 2.2929 & UGUCAGUUUGUCAAAUACCCCA \\
\hline mmu-miR-211 & 2.2731 & UUCCCUUUGUCAUCCUUUGCCU \\
\hline mmu-miR-31* & 2.2721 & UGCUAUGCCAACAUAUUGCCAUC \\
\hline mmu-miR-27a & 2.0945 & UUCACAGUGGCUAAGUUCCGC \\
\hline mmu-miR-3963 & 2.0212 & UGUAUCCCACUUCUGACAC \\
\hline mmu-miR-1a & 2.0141 & UGGAAUGUAAAGAAGUAUGUAU \\
\hline mmu-miR-3968 & 1.9957 & CGAAUCCCACUCCAGACACCA \\
\hline mmu-miR-200b & 1.9956 & UAAUACUGCCUGGUAAUGAUGA \\
\hline mmu-miR-200a* & 1.9578 & CAUCUUACCGGACAGUGCUGGA \\
\hline mmu-let-7b* & 1.9529 & CUAUACAACCUACUGCCUUCCC \\
\hline mmu-miR-3092 & 1.8662 & GAAUGGGGCUGUUUCCCCUCC \\
\hline mmu-miR-200b* & 1.8444 & CAUCUUACUGGGCAGCAUUGGA \\
\hline mmu-miR-21 & 1.8270 & UAGCUUAUCAGACUGAUGUUGA \\
\hline mmu-miR-205 & 1.7966 & UCCUUCAUUCCACCGGAGUCUG \\
\hline mmu-miR-203 & 1.7911 & GUGAAAUGUUUAGGACCACUAG \\
\hline mmu-miR-3072 & 1.7820 & UGCCCCCUCCAGGAAGCCUUCU \\
\hline mmu-miR-27a* & 1.7546 & AGGGCUUAGCUGCUUGUGAGCA \\
\hline mmu-miR-30e & 1.7120 & UGUAAACAUCCUUGACUGGAAG \\
\hline mmu-miR-28c & 1.6958 & AGGAGCUCACAGUCUAUUGA \\
\hline mmu-miR-1843b-5p & 1.6886 & AUGGAGGUCUCUGUCUGACUU \\
\hline mmu-miR-122 & 1.6574 & UGGAGUGUGACAAUGGUGUUUG \\
\hline mmu-miR-467d* & 1.6427 & AUAUACAUACACACACCUACAC \\
\hline mmu-miR-34c* & 1.6416 & AAUCACUAACCACACAGCCAGG \\
\hline mmu-miR-148a & 1.6200 & UCAGUGCACUACAGAACUUUGU \\
\hline mmu-miR-1298* & 1.5997 & CAUCUGGGCAACUGAUUGAACU \\
\hline mmu-miR-1949 & 1.5995 & CUAUACCAGGAUGUCAGCAUAGUU \\
\hline mmu-miR-15a & 1.5988 & UAGCAGCACAUAAUGGUUUGUG \\
\hline mmu-miR-497 & 1.5903 & CAGCAGCACACUGUGGUUUGUA \\
\hline mmu-miR-5127 & 1.5743 & UCUCCCAACCCUUUUCCCA \\
\hline mmu-miR-200c & 1.5620 & UAAUACUGCCGGGUAAUGAUGGA \\
\hline mmu-miR-467b* & 1.5491 & AUAUACAUACACACACCAACAC \\
\hline mmu-miR-205* & 1.5424 & GAUUUCAGUGGAGUGAAGCUCA \\
\hline mmu-miR-744* & 1.5254 & CUGUUGCCACUAACCUCAACCU \\
\hline mmu-miR-140 & 1.5219 & CAGUGGUUUUACCCUAUGGUAG \\
\hline mmu-miR-143 & 1.5215 & UGAGAUGAAGCACUGUAGCUC \\
\hline mmu-miR-19b & 1.5059 & UGUGCAAAUCCAUGCAAAACUGA \\
\hline mmu-miR-106b & 1.5015 & UAAAGUGCUGACAGUGCAGAU \\
\hline mmu-miR-335-5p & 1.5000 & UCAAGAGCAAUAACGAAAAAUGU \\
\hline
\end{tabular}

(E50K) transgenic retinal samples as compared to wild-type retinal samples, representing $10 \%$ of the 48 miRNAs represented on the array (Table I). Of these, 5 miRNAs passed the Volcano plot filtering screen to identify miRNAs that were expressed at significantly different levels in transgenic versus wild-type retinas ( $\mathrm{P}<0.05$; false discovery rate, $<0.05$; Fig. 1A). Each of these miRNAs was upregulated 2.32-3.20-fold in the retinas of OPTN (E50K) transgenic mice compared to wild-type mice (Fig. 1B). mmu-miR-141 was focused on as it exhibited the largest increase in expression in retinal samples 
from transgenic mice and RT-qPCR was used to validate the miRNA microarray results. Consistent with the microarray results, RT-qPCR analysis demonstrated that mmu-miR-141 was expressed at significantly higher levels in the retinas of transgenic mice compared to wild-type mice (Fig. 2).

Computationalprediction of potential targetgenes andnetwork analysis. Candidate target genes for mmu-miR-141 were identified using three commonly used prediction algorithms to reduce the unpredictable number of false positives: PicTar, miRanda and TargetScan. The results of the miRNA-mRNA regulatory network analysis indicated that mmu-miR-141 belonged to the miR- 8 family. The miR- 8 family also includes mmu-miR-200a, mmu-miR-200b, mmu-miR-200c and mmu-miR-429, all of which were upregulated in the retinas of OPTN (E50K) transgenic mice by microarray but did not achieve statistical significance.

\section{Discussion}

The OPTN E50K mutation is the only mutation currently confirmed to play a causative role in NTG pathogenesis (2). Transgenic mice that overexpress $O P T N$ E50K provide a model system for elucidating the molecular mechanisms leading to POAG (8). While the majority of previous studies have focused on direct protein-protein interactions, differences in the expression levels of core proteins have also been noted and may contribute to POAG pathogenesis. The mechanisms accounting for differential expression of proteins indicated in POAG are not clear.

miRNAs can regulate gene expression through sequence complementarity to the 3'-UTRs of target mRNAs. miRNA binding to target mRNAs can result in translational repression through mRNA degradation or translational inhibition (12). Accumulating evidence suggests that miRNAs act as novel cellular senescence regulators. However, there is little information regarding the potential involvement of miRNAs in the mediating effects originating from the expression of mutant proteins associated with disease processes, such as OPTN (E50K). The present results suggest that the miRNAs miR-141, miR-200a, miR-200b, miR-200c and miR-429, which all belong to the miR- 8 family, may be critical regulators of POAG induced by the OPTN (E50K) mutation.

The miR- 8 family has been demonstrated to regulate numerous biological processes, including the response to osmotic stress in zebrafish embryos (13) and the response to steroid signaling in Drosophila (14). miR-200b, miR-200c and miR-429 are $T B K 1$ gene-related miRNAs according to the miRbase database. The E50K mutation in OPTN may enhance its interaction with $T B K 1$ and affect OPTN function by rendering OPTN insoluble (15). However, no experiments have been performed to fully elucidate the mechanisms by which the OPTN (E50K) mutation induces POAG to date. Therefore, further research to understand the interaction of the OPTN (E50K) mutation with TBK1 could improve the management of POAG.

In conclusion, the present study identified a number of differentially expressed miRNAs in the retinas of OPTN (E50K) mutant transgenic mice. The results suggest several noteworthy directions for future research aimed at elucidating the role of miRNAs in glaucoma pathogenesis. Detailed analyses of miRNA expression patterns and the impact on retinal cell function may contribute to our understanding of the pathophysiological processes leading to POAG.

\section{Acknowledgements}

The present study was funded by the National Natural Science Foundation of China (grant no. 81271000) and the Major State Basic Research Development Program of China (973 Program, grant no. 2011CB707502). The authors thank Dr Peter Wilker for editing the manuscript.

\section{References}

1. Quigley HA and Broman AT: The number of people with glaucoma worldwide in 2010 and 2020. Br J Ophthalmol 90: 262-267, 2006.

2. Iwase A, Suzuki Y, Araie M, Yamamoto T, Abe H, Shirato S, Kuwayama Y, Mishima HK, Shimizu H, Tomita G, et al; Tajimi Study Group, Japan Glaucoma Society: The prevalence of primary open-angle glaucoma in Japanese: The Tajimi Study. Ophthalmology 111: 1641-1648, 2004.

3. Rezaie T, Child A, Hitchings R, Brice G, Miller L, Coca-Prados M, Héon E, Krupin T, Ritch R, Kreutzer D, et al: Adult-onset primary open-angle glaucoma caused by mutations in optineurin. Science 295: 1077-1079, 2002.

4. Aung T, Rezaie T, Okada K, Viswanathan AC, Child AH, Brice G, Bhattacharya SS, Lehmann OJ, Sarfarazi M and Hitchings RA: Clinical features and course of patients with glaucoma with the E50K mutation in the optineurin gene. Invest Ophthalmol Vis Sci 46: 2816-2822, 2005.

5. Leung YF, Fan BJ, Lam DS, Lee WS, Tam PO, Chua JK, Tham CC, Lai JS, Fan DS and Pang CP: Different optineurin mutation pattern in primary open-angle glaucoma. Invest Ophthalmol Vis Sci 44: 3880-3884, 2003.

6. Fuse N, Takahashi K, Akiyama H, Nakazawa T, Seimiya M, Kuwahara S and Tamai M: Molecular genetic analysis of optineurin gene for primary open-angle and normal tension glaucoma in the Japanese population. J Glaucoma 13: 299-303, 2004.

7. Alward WL, Kwon YH, Kawase K, Craig JE, Hayreh SS, Johnson AT, Khanna CL, Yamamoto T, Mackey DA, Roos BR, et al: Evaluation of optineurin sequence variations in 1,048 patients with open-angle glaucoma. Am J Ophthalmol 136: 904-910, 2003.

8. Chi ZL, Akahori M, Obazawa M, Minami M, Noda T, Nakaya N, Tomarev S, Kawase K, Yamamoto T, Noda S, et al: Overexpression of optineurin E50K disrupts Rab8 interaction and leads to a progressive retinal degeneration in mice. Hum Mol Genet 19: 2606-2615, 2010.

9. Xiao F, Zuo Z, Cai G, Kang S, Gao X and Li T: miRecords: An integrated resource for microRNA-target interactions. Nucleic Acids Res 37 (Database): D105-D110, 2009.

10. Luna C, Li G, Qiu J, Epstein DL and Gonzalez P: MicroRNA-24 regulates the processing of latent TGF $\beta 1$ during cyclic mechanical stress in human trabecular meshwork cells through direct targeting of FURIN. J Cell Physiol 226: 1407-1414, 2011.

11. Meng Q, Xiao Z, Yuan H, Xue F, Zhu Y, Zhou X, Yang B, Sun J, Meng B, Sun X, et al: Transgenic mice with overexpression of mutated human optineurin(E50K) in the retina. Mol Biol Rep 39: 1119-1124, 2012

12. Ambros V: MicroRNAs: Tiny regulators with great potential. Cell 107: 823-826, 2001

13. Flynt AS, Thatcher EJ, Burkewitz K, Li N, Liu Y and Patton JG: miR-8 microRNAs regulate the response to osmotic stress in zebrafish embryos. J Cell Biol 185: 115-127, 2009.

14. Jin H, Kim VN and Hyun S: Conserved microRNA miR-8 controls body size in response to steroid signaling in Drosophila. Genes Dev 26: 1427-1432, 2012.

15. Minegishi Y, Iejima D, Kobayashi H, Chi Z-L, Kawase K, Yamamoto T, Seki T, Yuasa S, Fukuda K and Iwata T: Enhanced optineurin E50K-TBK1 interaction evokes protein insolubility and initiates familial primary open-angle glaucoma. Hum Mol Genet 22: 3559-3567, 2013. 